\title{
Rotator cuff retear after repair surgery: comparison between experienced and inexperienced surgeons
}

\author{
Jin-Young Park ${ }^{1}$, Jae-Hyung Lee ${ }^{1}$, Kyung-Soo $\mathrm{Oh}^{2}$, Seok Won Chung ${ }^{2}$, Yunseong Choi ${ }^{1}$, Won-Yong Yoon ${ }^{1}$, \\ Dong-Wook Kim ${ }^{1}$
}

${ }^{1}$ Center for Shoulder, Elbow and Sports, Neon Orthopaedic Clinic, Seoul, Korea

${ }^{2}$ Department of Orthopaedic Surgery, Konkuk University School of Medicine, Seoul, Korea

\begin{abstract}
Background: We hypothesized in this study that the characteristics of retear cases vary according to surgeon volume and that surgical outcomes differ between primary and revision arthroscopic rotator cuff repair (revisional ARCR).

Methods: Surgeons performing more than 12 rotator cuff repairs (RCRs) per year were defined as high-volume surgeons, and those performing fewer than 12 RCRs were considered low-volume surgeons. Of the 47 patients who underwent revisional ARCR at our clinic enrolled in this study, 21 cases were treated by high-volume surgeons and 26 cases by low-volume surgeons. In all cases, the interval between primary surgery and revisional ARCR, degree of "acromial scuffing," number of anchors, RCR technique, retear pattern, fatty infiltration, retear size, operating time, and clinical outcome were recorded.

Results: During primary surgery, significantly more lateral anchors $(\mathrm{p}=0.004)$ were used, and the rate of use of the double-row repair technique was significantly higher $(\mathrm{p}<0.001)$ in the high- versus low-volume surgeon group. Moreover, the "cut-through pattern" was observed significantly more frequently among the cases treated by high- versus low-volume surgeons $(\mathrm{p}=0.008)$. The clinical outcomes after revisional ARCR were not different between the two groups.

Conclusions: Double-row repair during primary surgery and the cut-through pattern during revisional ARCR were more frequent in the high- versus low-volume surgeon groups. However, no differences in retear site or size, fatty infiltration grade, or outcomes were observed between the groups.
\end{abstract}

Keywords: Rotator cuff retear; Surgeon volume; Surgeon experience; High-volume surgeon; Low-volume surgeon; Arthroscopic rotator cuff repair

\section{INTRODUCTION}

Rotator cuff retear after arthroscopic rotator cuff repair (ARCR) significantly lowers the likelihood of a good clinical outcome and is therefore clinically important [1]. Rotator cuff retear rate ranges from $7.2 \%$ to $94 \%$ [2-5]. The risk factors for rotator cuff retear are larger initial tear size, older age, and degree of fatty degeneration $[6,7]$. The retear rate for ARCR is reported to be higher than that for primary ARCR [8]. Some studies have compared surgical outcomes according to surgeon volume $[9,10]$, but not for revisional ARCR. In this study, we hypothesized that the surgical findings and outcomes of revisional ARCR differ according to

Received: February 5, $2021 \quad$ Revised: June 4, $2021 \quad$ Accepted: June 7, 2021

Correspondence to: Dong-Wook Kim

Center for Shoulder, Elbow and Sports, Neon Orthopaedic Clinic, 8 Seolleung-ro 131-gil, Gangnam-gu, Seoul, 06059, Korea

Tel: +82-2660-7632, Fax: +82-2660-7599, E-mail: godkdurla@hanmail.net, ORCID: https://orcid.org/0000-0003-3831-5748

\section{Financial support: None.}

Conflict of interest: None.

Copyright@ 2021 Korean Shoulder and Elbow Society.

This is an Open Access article distributed under the terms of the Creative Commons Attribution Non-Commercial License (http://creativecommons.org/licenses/by-nc/4.0/) which permits unrestricted non-commercial use, distribution, and reproduction in any medium, provided the original work is properly cited. 
the surgical volume of the surgeon who performed the primary ARCR.

\section{METHODS}

This study was approved by the Institutional Review Board of Konkuk University Medical Center (IRB No. KUMC2019-11062). Informed consent was waived due to retrospective nature of this study.

\section{Patient Selection}

Of the 53 patients who underwent revisional ARCR from January 2009 to February 2019, this study included 47: three were excluded because their medical records did not include the primary surgeon, two because there were no arthroscopic images of the revision ARCR, and one because there was no preoperative magnetic resonance imaging (MRI) scan. The median age at the time of revisional ARCR was 61 years (range, 41-78 years). Thirty cases $(63.8 \%)$ involved the right shoulder and 17 (36.2\%) involved the left shoulder. The median follow-up period after revisional ARCR was 34 months (range, 18-78 months). Four of the 47 patients underwent open rotator cuff repair (RCR) and 43 underwent ARCR. The median interval between primary surgery and revisional ARCR was 20.5 months (range, 12-149 months).

\section{Shoulder Surgeon Volume}

The Health Insurance Review and Assessment Service was consulted to determine the number of RCR operations per year were conducted by each primary surgeon. The average number of RCRs per year was determined from the first year in which primary surgeons started to perform RCR onward. Weinheimer et al. [10] defined high-volume surgeons as those who performed at least 5 arthroplasties and at least 12 ARCRs per year. In this study, surgeons who performed 12 or more ARCRs per year were defined as high-volume surgeons and those who performed few- er than 12 as low-volume surgeons.

\section{Radiologic Assessment}

Preoperative MRI images were used to evaluate fatty infiltration, the number and location of anchors, the primary operative technique, and the retear pattern. Fatty infiltration was classified according to the highest Goutallier stage among the supraspinatus, infraspinatus, and subscapularis muscles [11]. Anchors were counted on the medial and lateral sides of the rotator cuff footprint. The primary surgical technique was considered as single-row when there were only medial anchors and as double-row when there were both medial and lateral anchors. In accordance with Cho et al. [12], the retear pattern was classified as type 1 when no rotator cuff tissue was visible in the rotator cuff footprint and as type 2 when remnant cuff tissue was present. The retear was classified as "extra only" if the retear did not affect the previous surgical area [13].

\section{Arthroscopic Finding}

All revisional ARCRs were performed by the senior surgeon (JYP) with the patient under general anesthesia in the beachchair position. All surgical findings were added to the patients' medical records, including photographs. Retear size was recorded to the millimeter based on arthroscopic images. The associations of radiologic findings, the primary RCR technique used, and the retear pattern were assessed. The degree of scuffing of the acromial undersurface (hereafter, "acromial scuffing") was evaluated using the Copeland-Levy classification (A0-A1, minor; A2-A3, major) (Fig. 1) [14]. The "cut-through pattern" is defined by the presence of tendon tissue torn longitudinally by the suture knot (Fig. 2) [15,16].

Clinical Assessment Preoperative status and postoperative outcomes were determined by reference to the medical records using a visual analog scale (VAS), the American Shoulder and Elbow Surgeons (ASES) score, the Korean Shoulder Scoring (KSS)
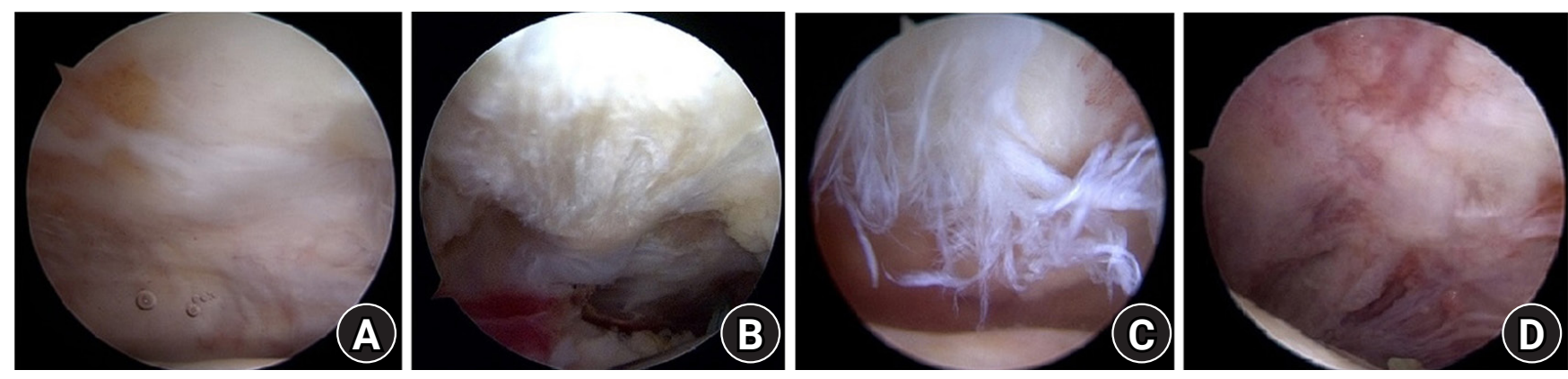

Fig. 1. Arthroscopic images: (A) no scuffing (A0), (B) minor scuffing (A1), (C) major scuffing and fibrillations (A2), and (D) acromial bare bone exposure (A3). 
system, and the Constant-Murley Score (CMS). Shoulder abduction power $(\mathrm{kg})$ was also recorded [17].

\section{Statistical Analysis}

Data are provided as mean and standard deviation and were checked for normality using the Kolmogorov-Smirnov test. All data, except the number of anchors and Goutallier stage, followed a normal distribution. Normal data were analyzed using paired and unpaired t-tests. Non-normally distributed data were analyzed using the Mann-Whitney U-test and Wilcoxon signedrank test. All analyses were performed with SPSS ver. 15.0 (SPSS

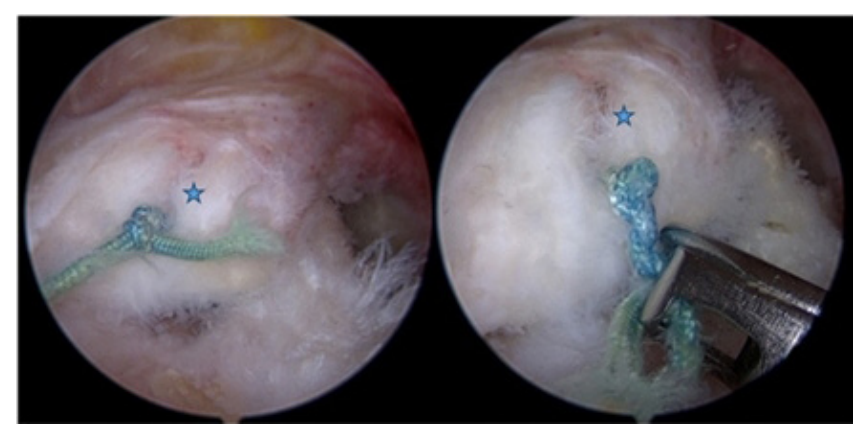

Fig. 2. Cuff tissue cut-through (asterisks).
Inc., Chicago, IL, USA). A p-value $<0.05$ was considered significant.

\section{RESULTS}

Of the 47 revisional ARCR cases, 21 surgeries were conducted by high-volume surgeons and 26 by low-volume surgeons. No differences in sex, age, or follow-up duration were observed between the high- and low-volume groups (Table 1). The time between primary and revision surgery $(\mathrm{p}=0.534)$, acromial scuffing $(p=0.853)$, number of medial anchors $(p=0.397)$, total number of anchors $(p=0.228)$, retear pattern (Rhee type) $(p=0.061)$, number of cases with extralesional tears only $(p=0.158)$, fatty infiltration (Goutallier stage) $(\mathrm{p}=0.284)$, retear size $(\mathrm{p}=0.545)$, and operating time for revision $(\mathrm{p}=0.528)$ were not different between the two groups. The number of lateral anchors and frequency of use of the double row operating technique were significantly higher in the high- versus low-volume group ( $\mathrm{p}=0.004$ and $p=0.000$, respectively). However, cases were omitted from the analysis if the repair technique could not be clearly identified based on the MRI or arthroscopic images. The cut-through pattern was observed more frequently in the high- versus low-volume group $(\mathrm{p}=0.008)$ (Table 2$)$.

Table 1. Demographics

\begin{tabular}{lccc}
\hline Variable & High-volume surgeon & Low-volume surgeon & p-value \\
\hline No. of patients & 21 & 26 & 0.165 \\
Sex (male:female) & $15: 6$ & $16: 10$ & 0.553 \\
Age (yr) & $62.1 \pm 7.2$ & $58.8 \pm 8.0$ & 0.668 \\
Follow-up (mo) & $42.1 \pm 22.5$ & $40.65 \pm 21.9$ & \\
\hline
\end{tabular}

Values are presented as mean \pm standard deviation.

Table 2. Rotator cuff retear characteristics

\begin{tabular}{lccc}
\hline Variable & High-volume surgeon & Low-volume surgeon & p-value \\
\hline Time from primary surgery (mo) & $37.2 \pm 36.4$ & $35.5 \pm 29.7$ & 0.534 \\
Major acromial scuffing & $10(47.4)$ & $12(46.2)$ & 0.853 \\
No. of medial anchors & $1.8 \pm 1.0$ & $1.6 \pm 1.2$ & 0.397 \\
No. of lateral anchor & $2.1 \pm 0.8$ & $1.5 \pm 1.2$ & $0.004^{*}$ \\
No. of total anchors & $3.9 \pm 1.6$ & $3.2 \pm 2.1$ & 0.228 \\
No. of single/double rows & $1 / 19^{\dagger}$ & $7 / 16^{\dagger}$ & $0.000^{*}$ \\
Cut-through & $5(23.8)$ & $2(7.7)$ & $0.008^{*}$ \\
Rhee type 1/2 & $(38.1) / 13(61.9)$ & $13(50 \%) / 13(50)$ & 0.061 \\
Extralesional tear only & $2(9.5)$ & $1(3.8)$ & 0.158 \\
Goutallier stage & $2.2 \pm 1.2$ & $2.2 \pm 1.2$ & 0.284 \\
Size of retear (mm) & $29.5 \pm 12.2$ & $27.8 \pm 13.5$ & 0.545 \\
Revision operation time (min) & $118.5 \pm 28.9$ & $122.6 \pm 20.8$ & 0.528
\end{tabular}

Values are presented as mean \pm standard deviation or number (\%).

${ }^{\star}$ Statistically significant; ${ }^{\dagger}$ Cases not clearly identified in magnetic resonance imaging and arthroscopic images were not included. 
The VAS, ASES, KSS, and CMS scores, range of motion, and abduction power were significantly different at the final follow-up after revision surgery compared to preoperatively (all $\mathrm{p}=0.000)$ (Table 3$)$. The preoperative VAS $(\mathrm{p}=0.545)$, ASES $(p=0.596)$, KSS $(p=0.556)$, and CMS scores $(p=0.714)$, range of motion (forward elevation, $\mathrm{p}=0.064$; external rotation, $\mathrm{p}=0.269$ ), and abduction power $(\mathrm{p}=0.077)$ were not significantly different between the high- and low-volume groups (Table 4). The postoperative VAS $(p=0.343)$, ASES $(p=0.099)$, KSS $(p=0.805)$, CMS scores $(p=0.100)$, range of motion $(p=0.199)$, and abduction power $(\mathrm{p}=0.402)$ were not significantly different between the high- and low-volume groups (Table 5).

Table 3. Revisional ARCR surgical outcomes

\begin{tabular}{|c|c|c|c|}
\hline Variable & Preoperative & Postoperative & p-value \\
\hline \multicolumn{4}{|l|}{ Range of motion $\left(^{\circ}\right)$} \\
\hline Forward elevation & $122.7 \pm 31.2$ & $135.8 \pm 14.6$ & $0.000^{*}$ \\
\hline External rotation & $45.6 \pm 22.9$ & $49.6 \pm 24.0$ & $0.000^{*}$ \\
\hline VAS score & $5.5 \pm 2.3$ & $3.2 \pm 2.1$ & $0.000^{*}$ \\
\hline ASES score & $48.7 \pm 21.5$ & $65.6 \pm 16.5$ & $0.000^{*}$ \\
\hline KSS system & $57.3 \pm 21.4$ & $70.8 \pm 13.2$ & $0.000^{*}$ \\
\hline CMS & $56.1 \pm 22.7$ & $73.0 \pm 10.0$ & $0.000^{*}$ \\
\hline Abduction power (kg) & $0.49 \pm 0.25$ & $0.66 \pm 0.39$ & $0.000^{*}$ \\
\hline
\end{tabular}

Values are presented as mean \pm standard deviation.

ARCR: arthroscopic rotator cuff repair, VAS: visual analog scale, ASES: American Shoulder and Elbow Surgeons, KSS: Korean Shoulder Scoring, CMS: Constant-Murley Score.

*Statistically significant.

Table 4. Revisional ARCR preoperative comparison

\begin{tabular}{lccc}
\hline Preoperative status & High-volume surgeon & Low-volume surgeon & p-value \\
\hline Range of motion $\left(^{\circ}\right)$ & & & 0.064 \\
$\quad$ Forward elevation & $116.7 \pm 41.4$ & $127.0 \pm 22.0$ & 0.269 \\
$\quad$ External rotation & $43.9 \pm 21.9$ & $46.8 \pm 23.9$ & 0.545 \\
VAS score & $5.1 \pm 2.1$ & $5.7 \pm 2.5$ & 0.596 \\
ASES score & $45.8 \pm 19.2$ & $50.4 \pm 23.2$ & 0.556 \\
KSS system & $52.2 \pm 19.2$ & $60.4 \pm 23.1$ & 0.714 \\
CMS & $56.5 \pm 21.9$ & $55.9 \pm 24.4$ & 0.077 \\
Abduction power $(\mathrm{kg})$ & $0.46 \pm 0.18$ & $0.51 \pm 0.29$ & \\
\hline
\end{tabular}

Values are presented as mean \pm standard deviation.

ARCR: arthroscopic rotator cuff repair, VAS: visual analog scale, ASES: American Shoulder and Elbow Surgeons, KSS: Korean Shoulder Scoring, CMS: Constant-Murley Score.

Table 5. Revisional ARCR postoperative comparison

\begin{tabular}{lccc}
\hline Postoperative outcome & High-volume surgeon & Low-volume surgeon & p-value \\
\hline Range of motion $\left(^{\circ}\right)$ & & & 0.187 \\
$\quad$ Forward elevation & $133.6 \pm 13.4$ & $137.5 \pm 15.6$ & 0.199 \\
$\quad$ External rotation & $49.2 \pm 21.3$ & $50.0 \pm 26.5$ & 0.343 \\
VAS score & $2.9 \pm 2.2$ & $3.29 \pm 1.5$ & 0.099 \\
ASES score & $61.6 \pm 22.2$ & $67.8 \pm 12.6$ & 0.805 \\
KSS system & $52.2 \pm 19.2$ & $60.4 \pm 23.1$ & 0.100 \\
CMS & $65.5 \pm 10.6$ & $77.5 \pm 6.7$ & 0.702 \\
Abduction power $(\mathrm{kg})$ & $0.49 \pm 0.33$ & $0.43 \pm 0.40$ & 0.402 \\
\hline
\end{tabular}

Values are presented as mean \pm standard deviation.

ARCR: arthroscopic rotator cuff repair, VAS: visual analog scale, ASES: American Shoulder and Elbow Surgeons, KSS: Korean Shoulder Scoring, CMS: Constant-Murley Score. 


\section{DISCUSSION}

Rotator cuff tears account for a significant proportion of all shoulder problems in the general population, and their prevalence increases with age [18]. A full-thickness rotator cuff tear, or a partial tear $>50 \%$, is an indication for RCR [19]. The open and arthroscopic surgical results of RCR have been reported to be $\operatorname{good}[2,20,21]$.

Several systematic reviews have assessed outcomes by surgeon volume. Weinheimer et al. [10] reported that surgery time, cost, complications, and length of stay increase when the volume of the shoulder surgeon is small. Shervin et al. [9] reported that the hip dislocation rate was lower after hip arthroplasty by high- versus low-volume surgeons; for other outcomes there was no difference by surgeon volume, or the results were inconclusive. A high frequency of double-row repairs during primary RCR and a higher frequency of the cut-through pattern were observed in the high-volume group in this study. No other outcomes differed between the surgeon volume groups.

No consensus has not been established regarding the indications or optimal surgical approach for acromioplasty [22]. We investigated whether the rate of performance of acromioplasty differed by surgical volume. However, there were insufficient data to determine the rate of performance of acromioplasty as the primary operation according to the extent of acromial scuffing. Studies have shown that the double-row repair technique confers benefits in terms of tendon healing and has a lower retear rate than single-row repair [23-25]. However, other studies showed no significant difference in clinical outcomes between the techniques for small-to-medium-sized cuff tears $[26,27]$.

There was a correlation toward a higher incidence of the Rhee type 2 retear pattern in our high- versus low-volume group $(p=0.061)$ [12]. This may be related to the large number of double row repairs performed by the high-volume surgeons. In cases with tears outside of the previous surgical site (extralesional only), the previous rotator cuff surgical site may be intact, which would tend to produce a more favorable result. However, further study is needed to confirm this.

No study has assessed the mechanism or risk factors for the cut-through rotator cuff retear pattern. The cut-through pattern leads to longitudinal tears in the medial part of the rotator cuff due to the suture anchors on footprint. Therefore, this pattern should not be used in cases of Rhee type 2 retear in which retear occurs in the medial aspect of the medial anchor. However, further study is needed to validate this [12].

In this study, many outcomes showed no difference by surgeon volume. Thus, even surgeons performing fewer than 12 shoulder surgeries per year could be considered for rotator cuff retear. An insufficient number of cases was a limitation of this study: the amount of data on certain outcomes, such as extralesional, Rhee type 2 , and cut-through tear patterns, was relatively small so further studies are needed. Also, no distinction was made between open and arthroscopic surgery for primary ARCR. In this study, we were interested in how the initial cuff tear size varied according to surgeon volume but could not obtain sufficient surgical records or MRI images of the primary surgeries to quantify the tear sizes. Thus, we tried to indirectly determine the sizes of the cuff tears according to the number of medial anchors used; although the number of medial anchors tended to higher in the high-versus low-volume group, the difference was not significant $(p=0.397)$. Furthermore, the number of anchors may have been counted inaccurately in some cases: biocomposite anchors used for primary ARCR are difficult to detect on MRI images, and some of the images were of insufficient quality to detect the anchors. Furthermore, some anchors have metal components which can impair image quality, while open repair can be performed without using any suture anchors.

Based on our results, the conclusion that all high-volume surgeons are distinctive to low-volume surgeons cannot be made. Although surgeon experience is an important factor, Guttman et al. [28] found no significant difference in surgical time after the first 10 cases. This finding suggests that not only the annual average number of operations, but also the cumulative number, is meaningful. Further study is needed to explore this issue. High-volume surgeons frequently employed double-row repair as the primary RCR technique, and the cut-through pattern was more prevalent in the high-versus low-volume group. However, there were no group differences in retear site or size, fatty infiltration grade, or any other outcome.

\section{ORCID}

Jin-Young Park https://orcid.org/0000-0002-3085-1354 Jae-Hyung Lee https://orcid.org/0000-0001-7359-9921 Kyung-Soo Oh https://orcid.org/0000-0002-9812-9130 Seok Won Chung Yunseong Choi https://orcid.org/0000-0002-8221-9289 https://orcid.org/0000-0002-1433-0743 Won-Yong Yoon1 Dong-Wook Kim https://orcid.org/0000-0002-3945-4961 https://orcid.org/0000-0003-3831-5748

\section{REFERENCES}

1. Yang J Jr, Robbins M, Reilly J, Maerz T, Anderson K. The clinical effect of a rotator cuff retear: a meta-analysis of arthroscopic 
single-row and double-row repairs. Am J Sports Med 2017;45: 733-41.

2. Galatz LM, Ball CM, Teefey SA, Middleton WD, Yamaguchi K. The outcome and repair integrity of completely arthroscopically repaired large and massive rotator cuff tears. J Bone Joint Surg Am 2004;86:219-24.

3. Tashjian RZ, Hollins AM, Kim HM, et al. Factors affecting healing rates after arthroscopic double-row rotator cuff repair. Am J Sports Med 2010;38:2435-42.

4. Sugaya H, Maeda K, Matsuki K, Moriishi J. Repair integrity and functional outcome after arthroscopic double-row rotator cuff repair. a prospective outcome study. J Bone Joint Surg Am 2007;89:953-60.

5. Frank JB, ElAttrache NS, Dines JS, Blackburn A, Crues J, Tibone JE. Repair site integrity after arthroscopic transosseous-equivalent suture-bridge rotator cuff repair. Am J Sports Med 2008;36:1496-503.

6. Lee YS, Jeong JY, Park CD, Kang SG, Yoo JC. Evaluation of the risk factors for a rotator cuff retear after repair surgery. Am J Sports Med 2017;45:1755-61.

7. Le BT, Wu XL, Lam PH, Murrell GA. Factors predicting rotator cuff retears: an analysis of 1000 consecutive rotator cuff repairs. Am J Sports Med 2014;42:1134-42.

8. Shamsudin A, Lam PH, Peters K, Rubenis I, Hackett L, Murrell GA. Revision versus primary arthroscopic rotator cuff repair: a 2-year analysis of outcomes in 360 patients. Am J Sports Med 2015;43:557-64.

9. Shervin N, Rubash HE, Katz JN. Orthopaedic procedure volume and patient outcomes: a systematic literature review. Clin Orthop Relat Res 2007;457:35-41.

10. Weinheimer KT, Smuin DM, Dhawan A. Patient outcomes as a function of shoulder surgeon volume: a systematic review. arthroscopy 2017;33:1273-81.

11. Somerson JS, Hsu JE, Gorbaty JD, Gee AO. Classifications in brief: goutallier classification of fatty infiltration of the rotator cuff musculature. Clin Orthop Relat Res 2016;474:1328-32.

12. Cho NS, Yi JW, Lee BG, Rhee YG. Retear patterns after arthroscopic rotator cuff repair: single-row versus suture bridge technique. Am J Sports Med 2010;38:664-71.

13. Choi CH, Kim SG, Nam JH. New retear pattern after rotator cuff repair at previous intact portion of rotator cuff. Clin Shoulder Elbow 2016;19:237-40.

14. Atoun E, Gilat R, van Tongel A, et al. Intraobserver and interobserver reliability of the Copeland-Levy classification for arthroscopic evaluation of subacromial impingement. J Shoulder Elbow Surg 2017;26:2167-72.

15. Lambrechts M, Nazari B, Dini A, et al. Comparison of the cheese-wiring effects among three sutures used in rotator cuff repair. Int J Shoulder Surg 2014;8:81-5.

16. Owens BD, Algeri J, Liang V, DeFroda S. Rotator cuff tendon tissue cut-through comparison between 2 high-tensile strength sutures. J Shoulder Elbow Surg 2019;28:1897-1902.

17. Tae SK, Rhee YG, Park TS, et al. The development and validation of an appraisal method for rotator cuff disorders: the Korean Shoulder Scoring System. J Shoulder Elbow Surg 2009;18: 689-96.

18. Fehringer EV, Sun J, VanOeveren LS, Keller BK, Matsen FA 3rd. Full-thickness rotator cuff tear prevalence and correlation with function and co-morbidities in patients sixty-five years and older. J Shoulder Elbow Surg 2008;17:881-5.

19. Matava MJ, Purcell DB, Rudzki JR. Partial-thickness rotator cuff tears. Am J Sports Med 2005;33:1405-17.

20. Gazielly DF, Gleyze P, Montagnon C. Functional and anatomical results after rotator cuff repair. Clin Orthop Relat Res 1994; (304):43-53.

21. Carr A, Cooper C, Campbell MK, et al. Effectiveness of open and arthroscopic rotator cuff repair (UKUFF): a randomised controlled trial. Bone Joint J 2017;99:107-15.

22. Ponzio DY, VanBeek C, Wong JC, et al. Profile of current opinion on arthroscopic acromioplasty: a video survey study. arthroscopy 2016;32:1253-62.

23. Hantes ME, Ono Y, Raoulis VA, et al. Arthroscopic single-row versus double-row suture bridge technique for rotator cuff tears in patients younger than 55 years: a prospective comparative study. Am J Sports Med 2018;46:116-21.

24. Hein J, Reilly JM, Chae J, Maerz T, Anderson K. Retear rates after arthroscopic single-row, double-row, and suture bridge rotator cuff repair at a minimum of 1 year of imaging follow-up: a systematic review. Arthroscopy 2015;31:2274-81.

25. Millett PJ, Warth RJ, Dornan GJ, Lee JT, Spiegl UJ. Clinical and structural outcomes after arthroscopic single-row versus double-row rotator cuff repair: a systematic review and meta-analysis of level I randomized clinical trials. J Shoulder Elbow Surg 2014;23:586-97.

26. Aydin N, Kocaoglu B, Guven O. Single-row versus double-row arthroscopic rotator cuff repair in small- to medium-sized tears. J Shoulder Elbow Surg 2010;19:722-5.

27. Baums MH, Kostuj T, Klinger HM, Papalia R. Rotator cuff repair: single- vs double-row: clinical and biomechanical results. Orthopade 2016;45:118-24.

28. Guttmann D, Graham RD, MacLennan MJ, Lubowitz JH. Arthroscopic rotator cuff repair: the learning curve. Arthroscopy 2005;21:394-400. 